\title{
Ethnicity and cardiovascular health inequalities in people with severe mental illnesses: protocol for the E-CHASM study
}

\author{
J. Das-Munshi ${ }^{1}$ - M. Ashworth ${ }^{2}$ F. Gaughran ${ }^{3}$ - S. Hull ${ }^{4}$ C. Morgan ${ }^{1}$ •

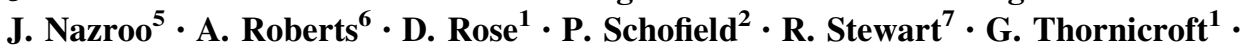 \\ M. J. Prince ${ }^{1}$
}

Received: 17 November 2015/ Accepted: 18 January 2016/Published online: 4 February 2016

(c) The Author(s) 2016. This article is published with open access at Springerlink.com

\begin{abstract}
Purpose People with severe mental illnesses (SMI) experience a 17 - to 20 -year reduction in life expectancy. One-third of deaths are due to cardiovascular disease. This study will establish the relationship of SMI with cardiovascular disease in ethnic minority groups (Indian, Pakistani, Bangladeshi, black Caribbean, black African and Irish), in the UK.

Methods E-CHASM is a mixed methods study utilising data from 1.25 million electronic patient records. Secondary analysis of routine patient records will establish if differences in cause-specific mortality, cardiovascular disease
\end{abstract}

J. Das-Munshi

jayati.das-munshi@kcl.ac.uk

1 Department of Health Service and Population Research, Centre for Epidemiology and Public Health, Institute of Psychiatry, Psychology and Neuroscience, King's College London, De Crespigny Park, PO 33, London SE5 8AF, UK

2 Division of Health and Social Care Research, Department of Primary Care and Public Health Sciences, King's College London, 3rd Floor, Addison House, Guy's Campus, London SE1 1UL, UK

3 South London and Maudsley Trust and King's College London, London, UK

4 Centre for Primary Care and Public Health, Blizard Institute, Queen Mary University of London, Yvonne Carter Building, 58 Turner Street, London E1 2AB, UK

5 University of Manchester, Manchester, England

6 Natural Language Processing Group, Department of Computer Science, University of Sheffield, Sheffield, England

7 Institute of Psychiatry, Psychology and Neuroscience, King's College London, Room M1.06, De Crespigny Park, London SE5 8AF, UK prevalence and disparities in accessing healthcare for ethnic minority people living with SMI exist. A nested qualitative study will be used to assess barriers to accessing healthcare, both from the perspectives of service users and providers. Results In primary care, 993,116 individuals, aged 18+, provided data from 186/189 (98\%) practices in four innercity boroughs (local government areas) in London. Prevalence of SMI according to primary care records, ranged from $1.3-1.7 \%$, across boroughs. The primary care sample included Bangladeshi $[n=94,643(10 \%)]$, Indian $[n=6086$ $(6 \%)]$, Pakistani $[n=35,596(4 \%)]$, black Caribbean $[n=45,013(5 \%)]$, black African $[n=75,454(8 \%)]$ and Irish people $[n=13,745(1 \%)]$. In the secondary care database, 12,432 individuals with SMI over 2007-2013 contributed information; prevalent diagnoses were schizophrenia $[n=6805(55 \%)]$, schizoaffective disorders $[n=1438$ $(12 \%)]$ and bipolar affective disorder $[n=4112(33 \%)]$. Largest ethnic minority groups in this sample were black Caribbean [1432 (12 \%)] and black African (1393 (11\%)).

Conclusions There is a dearth of research examining cardiovascular disease in minority ethnic groups with severe mental illnesses. The E-CHASM study will address this knowledge gap.

Keywords Severe mental illness - Ethnicity . Cardiovascular disease $\cdot$ Schizophrenia $\cdot$ Bipolar affective disorder

\section{Background}

People living with severe mental illnesses such as schizophrenia have a reduced life expectancy relative to the general population which is up to 20 years earlier in men and 17 years in women, in high income countries [1,2]. A 
large proportion of deaths are from chronic diseases, including coronary heart disease and stroke [3]. Increased mortality may be related to people with severe mental illnesses receiving poorer quality physical healthcare [4-6]. There is also a higher prevalence of metabolic risk factors such as obesity [7], hyperlipidaemia [8], diabetes [9] together with higher smoking rates [10], in these populations. Anti-psychotic medications, especially at higher doses, are associated with death from stroke and coronary heart disease [11]. People with severe mental illnesses experience barriers to seeking timely help for co-morbid medical problems [12]. Finally, there may be shared factors underlying premature mortality and severe mental illness, such as social disadvantage [12].

Health inequalities may be even more pronounced among ethnic minority populations with severe mental illnesses [13, 14]. The reasons for this are unclear. There is much evidence to suggest that those from ethnic minority groups may experience disadvantage within the mental healthcare system. Black people are more likely to be compulsorily detained [15], be less satisfied with prescribing [16] and more likely to be prescribed high-potency antipsychotics at high doses [17-19]. In addition, physical health monitoring may not be to the same standard as for white patients [20]. The prevalence of cardiovascular disease is known to be elevated in people living with severe mental illnesses and is also known to be of a greater concern for some ethnic minority groups [21]. The exact nature of the interaction between being of an ethnic minority background and living with severe mental illness, for the risk of cardiovascular disease-is less clear. Previous research examining cardiovascular disease health inequalities by ethnicity in severe mental illness populations has been limited by an over-reliance on small convenience samples recruited from clinics, with limited representativeness and without the inclusion of adequate numbers of people from ethnic minority groups to enable assessment of prevalence of cardiovascular disease [14].

The E-CHASM study (ethnicity and cardiovascular health inequalities in severe mental illness) described in this protocol will draw upon electronic health records from a large secondary care mental health Trust in England, as well as data from primary care, to enable analyses examining the mechanisms for premature mortality due to cardiovascular disease in ethnic minority people with severe mental illnesses. The catchment areas for the study (south east/east London) represent an ethnically and socioeconomically diverse part of London, typical of many innercities where ethnic minority communities reside and where the burden of chronic health conditions is greatest.

E-CHASM will additionally utilise an embedded qualitative study, with integration of qualitative findings with quantitative findings, adopting a mixed methods design [22]. Using this approach, it may be possible to understand trends revealed in quantitative data analysis, particularly from the perspectives of service users, carers and clinicians/service providers, which will help to elucidate mechanisms underlying quantitative findings.

\section{Objectives}

1. To understand the reasons for premature mortality in ethnic minority people living with severe mental illnesses; in particular to determine variations by ethnicity in the following:

(a) The effect of severe mental illness on cardiovascular risk factors;

(b) Cause-specific mortality patterns among people with severe mental illnesses;

(c) Quality of care received, relevant to premature mortality;

2. To develop and validate a measure for individuallevel socioeconomic position for application in a large secondary care electronic mental health records data resource, which will be used to improve the assessment of the association of self-ascribed ethnicity with health-related outcomes.

3. To assess barriers to equitable physical healthcare amongst ethnic minority people living with severe mental illnesses, from the perspectives of service users, their carers and clinicians.

\section{Hypotheses (for quantitative data analyses)}

Compared to white British people with severe mental illnesses, ethnic minority service users with severe mental illnesses will:

1. Have an elevated prevalence of cardiovascular disease risk factors (hypertension, diabetes, obesity, raised serum cholesterol, and smoking) and be less likely to have had these adequately managed, as determined by national standards for clinical management.

2. Be less likely to have cardiovascular disease risk factors adequately screened or managed, when prescribed neuroleptic medication;

3. Be more likely to be prescribed multiple antipsychotics or antipsychotics at higher doses or outside recommended dose ranges.

4. Experience differing causes of mortality (i.e., causespecific mortality fractions), in particular excess risk of mortality due to coronary artery disease. 


\section{Qualitative study aims}

The aim of the qualitative phase of this study is to identify barriers to delivering equitable physical healthcare (from clinician perspectives) or in accessing healthcare (service user and/or carer perspectives).

\section{Qualitative study questions}

1. What are the barriers to having physical health monitored and treated in black and minority ethnic people living with severe mental illness?

2. How are physical health problems related to cardiovascular disease managed by black and minority ethnic participants living with severe mental illnesses?

\section{Methods}

\section{Design}

To address key objectives, this study will utilise anonymised patient data from a variety of sources, some of which will be linked together (e.g., secondary care patient data linked to Office for National Statistics (ONS) mortality data). This programme of research will follow a quantitative study design leading on to a nested qualitative study. In the final phase, results from the quantitative and qualitative studies will be integrated.

\section{Quantitative research methodology}

\section{Overview of data from primary care}

Setting The London boroughs represented in the study (Lambeth, Tower Hamlets, Newham, City and Hackney) are notable for being home to the largest ethnic minority communities in the UK, including Bangladeshi, black Caribbean and black African communities; up to $51 \%$ in these areas comprise people of an ethnic minority background [23]. These areas are also characterised by high population density and poverty [23, 24]. Tower Hamlets, Newham and Hackney have the highest levels of deprivation in England [25]. The location of the study is characteristic of many other urban locations where ethnic minority communities reside within the UK [24].

Measures from primary care The quality and outcomes framework (QOF), a pay-for-performance scheme [26] introduced into primary care in the UK in 2004 [27], ensures that data quality is good for key indicators of health [28-30]. Information from primary care records will be extracted through data entered into structured fields in primary care electronic patient records (Read Codes [31]). Healthcare records contain information on consultation rates, clinical measurement values, prescribing and healthscreening [28-30].

\section{Demographic indicators}

Information on patient age, gender and ethnicity will be collected for analyses. Data will be linked to indices for area-level deprivation, such as the index of multiple deprivation [32], at small geographic level.

\section{Severe mental illnesses}

General practitioners are financially incentivised to maintain a register of people with severe mental illnesses. Individuals with a diagnosis of schizophrenia, bipolar affective disorder or non-organic psychosis, comprise people on this register [31]. The use of computer-based electronic records to identify patients with severe mental illnesses in primary care has previously been validated, with a sensitivity of $91 \%$ and positive predictive value of $91 \%$ for non-organic psychosis, assessed against a syndrome checklist derived from the Present State Examination and International Classification of Disease-9 (ICD-9) [33], applied to clinical case notes [34]. Recent work has shown that these diagnostic groupings remain stable over time [35].

\section{Cardiovascular disease indicators}

Diagnostic read codes will be used to ascertain presence of main cardiovascular health indicators. Diabetes mellitus, hypertension, weight (body mass index), smoking status and presence of hyperlipidaemia will be ascertained by presence of diagnostic codes, blood test results and other measurements (e.g., glycated haemoglobin (HbA1c), lipid profile readings, etc.)

\section{Psychotropic medications}

Information on prescriptions of antipsychotic medications according to formulation (oral/depot) and dose, will be extracted and classified [36] prior to analyses.

\section{Overview of data from secondary care}

South London and Maudsley NHS Foundation Trust Biomedical Research Centre (SLaM BRC) Case Register

This is an anonymised data resource drawn from the electronic health records of over 250,000 service users who 
have received care from a large secondary mental health service provider organisation (South London and Maudsley NHS Foundation Trust)37. South London and Maudsley is one of the largest mental health Trusts in Europe serving a base population of approximately 1.2 million people [37] living in south east London and using electronic health records across all its services since 2006 (with some services adopting these earlier). This data source contains all patient contacts with Trust services including out-patient appointments and in-patient admissions. The information from the electronic health record is accessed via the Clinical Record Interactive Search (CRIS) software system, which permits free text and structured fields to be searched for relevant information regarding patient characteristics, interventions and outcomes. All data since 2007 will be used in the E-CHASM study.

\section{Measures from secondary care}

\section{Demographic indicators}

Information on age, gender and self-ascribed ethnicity will be used. Area-level deprivation indicators matched to the last census will be used in initial analyses; however, work over the course of this study will lead to the derivation of a variable for individual-level socioeconomic position, as detailed below.

\section{Psychotropic medications}

Detail on antipsychotic medications will be derived from the health records and classified into formulation (oral/ depot) and dose [36]. Information on medications prescribed will be extracted from structured fields. In order to minimise missing data on prescribed medications, natural language processing with a bespoke algorithm will also be used to extract relevant information on prescribing from free text [38].

\section{Mental disorder}

Within South London and Maudsley Trust (SLaM), clinical teams are required to assign mental disorder diagnoses for all service users [37]. Diagnoses are entered into structured fields on the electronic record. Due to auditing of diagnosis, completion rates for diagnostic fields are high [37]. Using information from these fields, supplemented by natural language processing of free text and clinical note fields [38], the following ICD-10 diagnostic groups will be included in analyses: schizophreniform disorders (F20 F29), mania and bipolar affective disorder (F30, F31).

\section{Cause-specific mortality}

All electronic patient records contained within this dataset have a unique NHS patient identifier which can be linked to death certificate information nationally. Lists of deceased patients are downloaded on a monthly basis from the NHS care records service. To ascertain cause of death, information from linked death certificates will be extracted and categorised according to ICD-10 [39].

\section{Development of a measure for individual-level socioeconomic position}

Much important information is captured within the freetext information fields of electronic patient records [40, 41], particularly in mental healthcare. Using computational techniques such as natural language processing may help to unlock this information from within these text fields [41]. Within the SLaM-BRC Case Register, this has already been applied to derive information on cognition [42], smoking [43], pharmacotherapy [38] and symptoms [44]. The software [General Architecture for Text Engineering (GATE)] is an open source package used for natural language processing [45]. For example, in a study designed to assess smoking use, natural language processing supplemented information within structured fields, leading to an increase in proportions identified as smokers, from $11.6 \%$ (when reliant on structured fields alone) to $64 \%$ (when supplemented by natural language processing of free text) [43]. This approach is robust and repeatable [43]. Algorithms using GATE will be developed to derive indicators for socioeconomic position, through first ascertaining a priori keywords indicative of socioeconomic position, followed by an iterative process of: (1) developing a bespoke gazetteer of terms related to socioeconomic position (together with synonyms), and rules and models for extracting socioeconomic position; (2) evaluating the real world use and applicability of these terms and rules in routine clinical records. At least two indicators of socioeconomic position will be derived: education and occupational social class. Education is a valuable measure of socioeconomic position as it reflects early life socioeconomic position and is strongly related to parental characteristics [46]. Occupational social class is an important measure of socioeconomic position as it taps into individual social standing, conditions relating to work-based stress, and is also predictive of income and material resources [46]. Both have clear associations with mental and physical health $[46,47]$. 
Table 1 Smallest effect sizes (odds ratios) detectable for the largest and smallest ethnic minority groups at $80 \%$ power (with two sided $5 \%$ significance levels), for exposures with a prevalence of 10,20 and $50 \%$ in the reference (white British) group

\begin{tabular}{|c|c|c|c|c|}
\hline \multirow{2}{*}{$\begin{array}{l}\text { Primary care data source } \\
\text { White British }\end{array}$} & \multirow{2}{*}{$\begin{array}{l}N \\
238,211\end{array}$} & \multicolumn{3}{|c|}{ Prevalence of outcome in white British group } \\
\hline & & $10 \%$ & $20 \%$ & $50 \%$ \\
\hline \multicolumn{5}{|l|}{ Smallest detectable odds ratio } \\
\hline Bangladeshi & 93,143 & 1.03 & 1.02 & 1.01 \\
\hline Irish & 13,459 & 1.08 & 1.05 & 1.03 \\
\hline Secondary care data source & $N$ & \multicolumn{3}{|c|}{ Prevalence of outcome in white British group } \\
\hline white British & 28,618 & $10 \%$ & $20 \%$ & $50 \%$ \\
\hline \multicolumn{5}{|l|}{ Smallest detectable odds ratio } \\
\hline 'Other' white & 4477 & 1.14 & 1.09 & 1.05 \\
\hline Indian & 711 & 1.35 & 1.23 & 1.11 \\
\hline
\end{tabular}

'Poor quality' of care will be deemed present when therapeutic interventions fall outside of nationally recognised guidelines, in the UK. This might include a failure to adequately screen and manage physical health comorbidities (as detected in primary care data sources), or in the prescribing of antipsychotic medications in excess of recommended dose. Multivariable logistic regression will assess the association of ethnicity (with 'white British' as the reference) with each of the care indicators, adjusting or stratifying by age and gender, as appropriate.

4. Validation of the measure for individual-level socioeconomic position, derived using structured field information and natural language processing.

The construct and concurrent validity of the GATEderived socioeconomic position indicators of education and occupational social class will be assessed using structural equation modelling against a nested cohort of 558 individuals with psychosis [50]. These individuals presented to South London and Maudsley Trust services with a first episode of psychosis between 1st May 2010 and 30th April 2012. Individuals within this cohort were aged 18-64. Information on education and occupation were extracted from patient records by research workers, using the Medical Research Council (MRC) socio-demographic schedule [51].

\section{Sample size calculation}

The following table details detectable effect sizes at $80 \%$ power. There is greater power to detect associations in the primary care sample for each of the ethnic groups, as it is a larger sample (Table 1).

\section{Qualitative study}

\section{Methodology}

To address the objectives for the qualitative work, focus groups and interviews will be conducted with patients and 
clinicians. Focus groups stimulate discussion and involve group processes that can help people to explore and clarify views and provide insight into cultural values and norms. Up to eight focus groups, each comprising 6-8 participants will be facilitated by two interviewers, with one group comprising clinicians and at least one group containing individuals who identify their ethnicity as "white British'. In practice, the actual number of focus groups to be conducted will be determined by the range of relevant characteristics that emerge from the quantitative work and how these might best be reflected and balanced within the focus groups [52]. The purpose of the groups will be to understand the perspectives of service users and their carers, in particular experiences of living with cardiovascular disease co-morbid with severe mental illnesses and to identify barriers as well as facilitators to accessing healthcare.

Individual qualitative interviews will then be conducted to further explore the personal experience and relevance of themes identified from the focus groups. It is envisaged that up to twenty individual interviews will be conducted for this purpose.

Purposive sampling will be used to identify participants for both the focus groups and the individual interviews. The criteria for this will be determined after examination of the quantitative data (which may for example give an indication of which ethnic minority groups with severe mental illnesses experience physical health inequalities). This process is described in more detail next.

\section{Sampling frame for qualitative research}

For people living with severe mental illness, the 'Consent for Contact' programme [47], a register of South London and Maudsley Trust service users who are willing to be contacted about research projects on the basis of information in their record, will be used to purposively sample participants who have a severe mental illness co-morbid with a known physical health condition. To date, of a total of 9564 service users approached to take part in the 'Consent for Contact' programme, $72 \%$ have consented to being contacted for mental health research. There are approximately 1340 individuals with severe mental illnesses, who will form the main pool of participants to be approached to take part in focus groups and interviews for the qualitative part of the study. As this register is linked to the electronic patient record data source detailed above, purposive sampling will be based on characteristics considered important for the composition of the groups (e.g., ethnicity and/or presence of a physical health problem). The composition of focus groups will be informed by findings from the quantitative analyses, which will highlight where inequalities with cardiovascular health in people living with severe mental illness are most pronounced. Stratification [52] will be used to ensure a diversity of people representative of the population are included in the study, dependent on the types of question generated by quantitative findings and may for example include people living with severe mental illnesses who have also been diagnosed with type 2 diabetes mellitus.

\section{Topic guide for qualitative study}

Topic guides will be semi-structured. Domains to be covered in the topic guide will include: experiences of accessing/using mental and physical healthcare, the use of alternative therapies and medical models, experiences of physical symptoms, use of biomedical treatments and disease monitoring, perceptions of stigma/discrimination from health service providers and individuals. Other domains will include perceived barriers to care, including language, recent migration and knowledge of local services. The topic guide will be further developed following feedback from service user representatives.

\section{Analysis of qualitative data and integration with quantitative findings}

Thematic content analysis will be used to identify salient themes, until no further themes emerge. Analyses will be iterative as emergent themes will be used to generate hypotheses which may be tested in the quantitative dataset. Coding of qualitative data will be through appropriate software (N-Vivo) [53]. Emergent themes and coding frameworks will be cross-checked with researchers with expertise in qualitative research. Findings from the qualitative phase will be triangulated with those from the quantitative phase. A triangulation protocol will be used to identify 'meta-themes' relevant to findings from both quantitative and qualitative phases of the study [54] and in particular, areas of convergence or divergence.

\section{Ethical standards}

All data will be anonymised and managed according to UK National Health Service (NHS) information governance (IG) requirements.

Ethical approval to examine data from the South London and Maudsley Trust Biomedical Research Centre Case Registry (SLaM BRC case registry) as an anonymised dataset for secondary analysis has been obtained from 
Table 2 Characteristics of primary care database, sample restricted to $18+$

\begin{tabular}{|c|c|c|c|c|}
\hline & Lambeth & Tower Hamlets & Newham & City and Hackney \\
\hline Participants N (\%) & $295,516(30)$ & $214,600(21.6)$ & $282,512(28.4)$ & $200,488(20.2)$ \\
\hline Practices $N(\%)$ & $47(25)$ & $37(20)$ & $64(34)$ & $41(22)$ \\
\hline Proportion resident in most deprived $\operatorname{areas}^{\mathrm{a}} N(\%)$ & $254,593(86.2)$ & $169,036(90.0)$ & $263,681(98.8)$ & $176,463(97.7)$ \\
\hline Proportion ethnic minorities $N(\%)$ & $152,307(62.1)$ & $133,727(68.2)$ & $220,549(84.0)$ & $118,637(66.9)$ \\
\hline \multicolumn{5}{|l|}{ Prevalence severe mental illness } \\
\hline$N$ & 4718 & 3477 & 3706 & 3484 \\
\hline$\%(95 \% \mathrm{CI})$ & $1.60(1.55,1.64)$ & $1.62(1.57,1.67)$ & $1.31(1.27,1.35)$ & $1.74(1.68,1.80)$ \\
\hline \multicolumn{5}{|l|}{ Prevalence type 2 diabetes } \\
\hline$N$ & 13,372 & 13,479 & 20,309 & 10,754 \\
\hline$\%(95 \% \mathrm{CI})$ & $4.54(4.47,4.62)$ & $6.30(6.20,6.40)$ & $7.20(7.11,7.30)$ & $5.38(5.28,5.48)$ \\
\hline \multicolumn{5}{|l|}{ Prevalence hypertension } \\
\hline$N$ & 32,454 & 22,238 & 38,632 & 24,993 \\
\hline$\%(95 \% \mathrm{CI})$ & $10.98(10.87,11.10)$ & $10.36(10.23,10.49)$ & $13.67(13.55,13.80)$ & $12.47(12.32,12.61)$ \\
\hline \multicolumn{5}{|l|}{ Prevalence ischaemic heart disease } \\
\hline$N$ & 5103 & 4792 & 6759 & 4226 \\
\hline$\%(95 \% \mathrm{CI})$ & $1.73(1.68,1.77)$ & $2.23(2.17,2.30)$ & $2.39(2.34,2.45)$ & $2.11(2.05,2.17)$ \\
\hline \multicolumn{5}{|l|}{ Current or ex-smoker } \\
\hline$N$ & 132,741 & 48,266 & 47,749 & 46,412 \\
\hline$\%(95 \% \mathrm{CI})$ & $46.62(46.44,46.80)$ & $50.40(50.08,50.72)$ & $40.31(40.02,40.59)$ & $50.33(50.00,50.65)$ \\
\hline
\end{tabular}

${ }^{a}$ Bottom two quintiles for index of multiple deprivation 2000 at lower super output level; prevalence estimates based on number of patients on quality and outcomes framework $(\mathrm{QoF})$ registers for each disease, crude estimates

Oxfordshire REC C in 2008 and renewed in 2013 (reference number 08/H0606/71+5). Methods to de-identify data have been published and are robust [55]. Separate approvals to conduct the analyses proposed within this protocol have been granted by the CRIS Oversight Committee.

The South London Primary Care Research Governance Team reviewed the process of anonymised data analysis for patient data from Lambeth and approved the usage of aggregated anonymised patient data for research purposes. Lambeth Clinical Commissioning Group, Information Governance Steering Group (Lambeth CCG IGCG) has to approve each research project based on individual applications using a standardised proforma, the 'Privacy Impact Statement'.

For data from east London (Newham, Tower Hamlet, City and Hackney), each of the practices opted into the study by signing forms permitting the Clinical Effectiveness Group (CEG) to use anonymised aggregated data for audits and research projects supported by the CEG. Information Technology (IT) information governance committees provided approval for each of the three localities covered by the CEG.

Qualitative study Separate ethical approval will be sought for the qualitative part of the study once the topic guide and likely composition of focus groups have been finalised, following completion of initial analyses of quantitative data.

\section{Results}

\section{Primary care}

The primary care database comprises approximately 1.06 million patient records, including 358,614 anonymised electronic patient records registered to 47 (of 48) general practices in Lambeth and 697,600 anonymised records of patients registered to 142 (of 144) general practices in East London (Tower Hamlets, Newham, City and Hackney) (in total $98.3 \%$ of practices). Data on age and gender are near complete, since these are recorded routinely at patient registration. Self-ascribed ethnicity according to Office for National Statistics (ONS) census categories is available for $80-90 \%$ of patients, following local schemes to improve the recording of ethnicity $[28-30,56]$.

People registered to general practitioners/family doctors within the primary care database are more likely to reside in areas which are deprived and a high proportion of residents in each of the boroughs report their ethnicity as being of minority status (Table 2). Prevalence of severe mental illnesses ranges from $1.3-1.7 \%$, by borough 


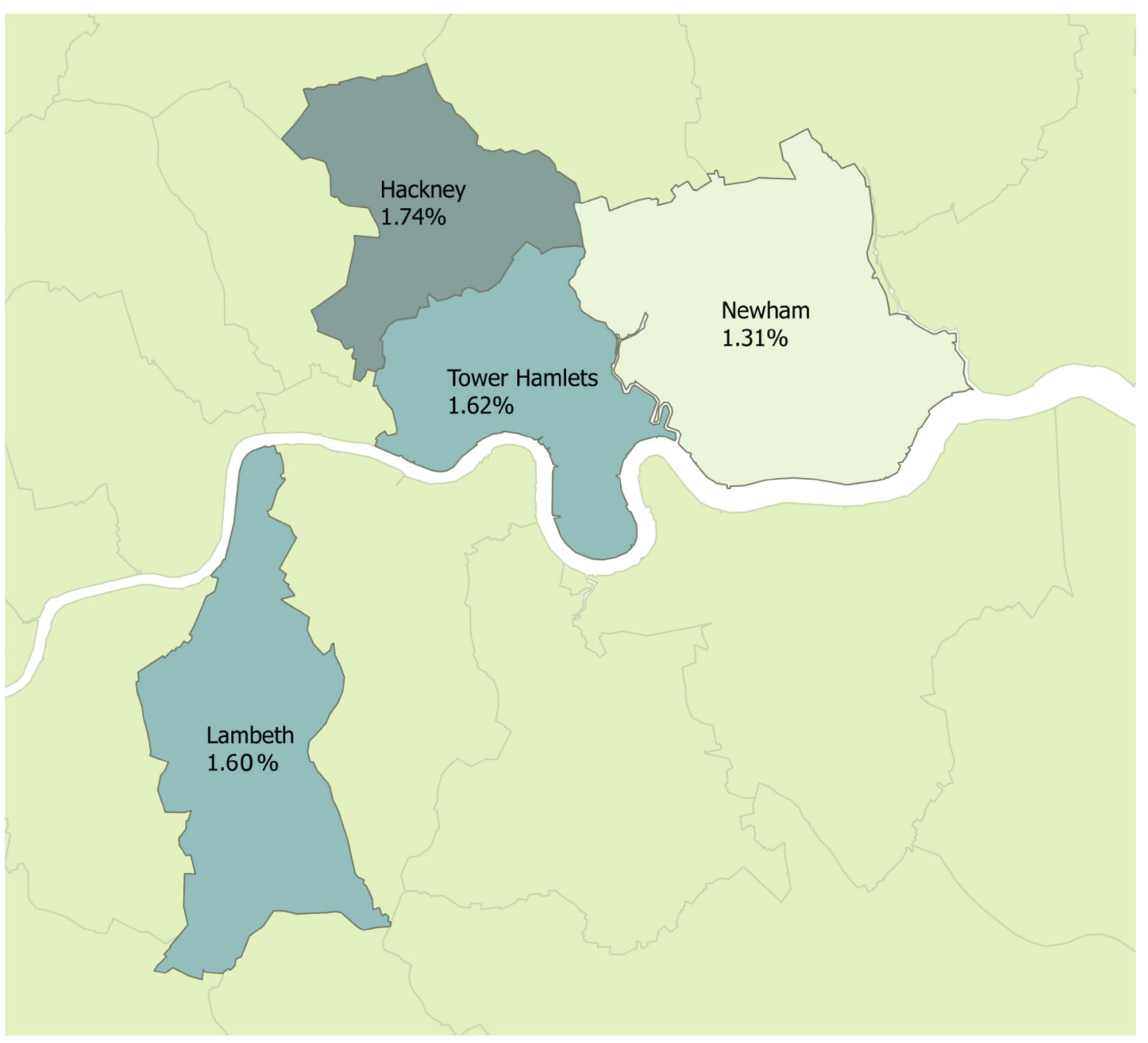

Fig. 1 Prevalence of severe mental illnesses across the study sites, by borough

(Table 2). Figure 1 highlights the geographical distribution of severe mental illness across the study sites.

Compared to people registered to general practitioners and not known to have a severe mental illness, the severe mental illness sample in primary care were older, with a higher proportion of men, a larger proportion of people reporting their ethnicity as white British or black Caribbean and were more likely to reside in deprived areas (Table 3).

\section{Secondary care}

The secondary care database currently comprises approximately 260,000 anonymised patient records [37], and has increased consistently by around 20,000 per year. Age and gender are complete in this database. Ethnicity is selfascribed according to standardised criteria, consistent with the last census. Information on self-ascribed ethnicity was 93-97\% complete in 2007-2013. Table 4 displays ICD-10 diagnoses for severe mental illnesses by ethnicity, for this database.

\section{Discussion}

People living with severe mental illnesses experience a dramatic reduction in life expectancy [1], a large proportion is accounted for through cardiovascular disease [3]. Although complex [57], a parallel body of work has highlighted the particular problem of cardiovascular disease for many ethnic minority groups [21, 58, 59]. It is, therefore, surprising that there is a dearth of evidence, relating to cardiovascular disease in ethnic minority groups living with severe mental illnesses [14]. This is a concern, as this represents preventable causes of death. The present study, E-CHASM, will address this gap in knowledge. 
Table 3 Characteristics of people living with severe mental illnesses in primary care database, sample restricted to adults aged $18+$

\begin{tabular}{|c|c|c|c|c|c|}
\hline & \multicolumn{2}{|c|}{$\begin{array}{l}\text { Not on SMI register } \\
N=997,731\end{array}$} & \multicolumn{2}{|c|}{$\begin{array}{l}\text { Severe mental illness (SMI register) } \\
N=15,385\end{array}$} & \multirow{2}{*}{$\begin{array}{l}\text { Total } \\
N\end{array}$} \\
\hline & $N$ & $\%$ & $N$ & $\%$ & \\
\hline Age (mean, SD) & $40(15.5)$ & & $47(15.3)$ & & 993,116 \\
\hline \multicolumn{6}{|l|}{ Sex } \\
\hline Men & 494,304 & 51 & 8488 & 55 & 502,792 \\
\hline Women & 483,426 & 49 & 6897 & 45 & 490,323 \\
\hline \multicolumn{6}{|l|}{ Ethnicity } \\
\hline White British & 238,211 & 27 & 4403 & 31 & 242,614 \\
\hline Irish & 13,459 & 2 & 286 & 2 & 13,745 \\
\hline 'Other' white & 171,493 & 20 & 1442 & 10 & 172,935 \\
\hline Indian & 60,298 & 7 & 566 & 4 & 60,864 \\
\hline Pakistani & 35,215 & 4 & 381 & 3 & 35,596 \\
\hline Bangladeshi & 93,143 & 11 & 1500 & 10 & 94,643 \\
\hline Black Caribbean & 43,367 & 5 & 1646 & 11 & 45,013 \\
\hline Black African & 74,037 & 9 & 1417 & 10 & 75,454 \\
\hline 'Other'/Chinese & 110,094 & 13 & 2027 & 14 & 112,121 \\
\hline Mixed ethnicity & 27,877 & 3 & 717 & 5 & 28,594 \\
\hline \multicolumn{6}{|c|}{ Area-level deprivation (quintiles) } \\
\hline 1 Most deprived & 587,820 & 64 & 10,437 & 71 & 589,257 \\
\hline 2 & 261,966 & 29 & 3550 & 24 & 265,516 \\
\hline 3 & 51,207 & 6 & 521 & 4 & 51,728 \\
\hline 4 & 10,396 & 1.1 & 96 & 0.7 & 10,492 \\
\hline 5 Least deprived & 4626 & 0.5 & 21 & 0.1 & 4647 \\
\hline
\end{tabular}

a Severe mental illness refers to patients with any of schizophrenia, schizoaffective disorder, bipolar affective disorder and any non-organic psychosis

$p<0.001$ for all sociodemographic variables; comparing SMI to non-SMI group

\begin{tabular}{lccc}
\hline & $\begin{array}{l}\text { Schizophrenia } \\
N=6885\end{array}$ & $\begin{array}{l}\text { Schizoaffective disorder } \\
N=1438\end{array}$ & $\begin{array}{l}\text { Bipolar affective disorder } \\
N=4112\end{array}$ \\
\hline Ethnicity, $n(\%)$ & & & \\
White British & $2271(33)$ & $519(36)$ & $2033(49)$ \\
'Other' white & $473(7)$ & $93(6)$ & $376(9)$ \\
Irish & $141(2)$ & $37(3)$ & $132(3)$ \\
Indian & $131(2)$ & $21(1)$ & $64(2)$ \\
Pakistani & $55(0.1)$ & $10(0.7)$ & $24(0.6)$ \\
Bangladeshi & $29(0.4)$ & $8(0.6)$ & $18(0.4)$ \\
Black Caribbean & $1004(15)$ & $173(12)$ & $255(6)$ \\
Black African & $926(13)$ & $194(13)$ & $606(15)$ \\
'Other'/Chinese & $1472(21)$ & $284(20)$ & $48(1)$ \\
Mixed ethnicity & $97(1)$ & $22(1.5)$ & $257(6)$ \\
Not stated/missing & $286(4)$ & $71(4.9)$ & \\
\hline
\end{tabular}

Table 4 Breakdown of severe mental illness diagnosis by ethnicity in secondary care database, 2007-2013 illness are, however, broadly consistent with those published by Public Health England [59]. Cardiovascular disease indicators presented here are broadly consistent with prevalence estimates published for London [60].

The feasibility of E-CHASM rests on its usage of routine electronic patient records to establish differences in

\section{to $0.8 \%$, with considerable variability between geographi-}


prevalence and treatment access. In particular, analyses of records from patients registered to general practices in an ethnically and socioeconomically diverse region in a major inner-city conurbation, alongside analysis of records from a large mental health Trust serving these populations, will enhance the study. The enriched representation of populations normally under-represented or absent in similar work [14] will allow the assessment of ethnic minority physical health inequalities in severe mental illness populations. Methodological techniques such as natural language processing to data-mine free text within secondary care records [41, 45] will enable development of measures for socioeconomic position through robust and repeatable methods which will also enable the automation of checking of a large volume of records which would otherwise be impossible [41]. As far as we are aware, the derivation of an individual-level measure for socioeconomic position from routine electronic health records has not been previously attempted. Finally a novel application of using electronic patient records in research is in the application of the 'consent for contact' programme at South London and Maudsley Trust. Thus, following analysis of quantitative data, it will be possible to purposively sample potential participants who will be invited to take part in focus groups and individual interviews for the qualitative phase of this study, based on important attributes (e.g., type of diagnosis, ethnicity, presence of physical comorbidity). Thus, the findings from the quantitative phase will directly inform qualitative data collection. This form of integration, known as 'connecting', will draw from the strengths of deductive methods in the quantitative phase to inform study design for the qualitative phase [22]. Integration of findings across qualitative and quantitative data sources [54] will help to understand mechanisms underlying quantitative findings as well as identify barriers to care from the perspectives of service users, carer and service providers. A future application of this data source could be to assess discrepancies in care provided across primary and secondary care. This would be based on data linkages between primary and secondary care, which could be explored in future work.

\section{Strength and limitations}

Strengths of this study include the power to conduct statistical analyses in groups of individuals who form a minority in the population and thus address the current scarcity of research in this field. Other strengths include the usage of natural language processing to derive a measure for individual-level socioeconomic position. If successful, this will provide a methodological advantage, as almost all previous work using electronic health records has tended to rely on area-level measures for deprivation. The mixed methods design of the study will enhance possibilities of understanding trends in quantitative analyses as well as highlighting barriers to equitable care from the perspectives' of service users and service providers. In all of the quantitative data sources, ethnicity is self-ascribed. This is an addition over previous work which has tended to rely on country of birth [61].

Limitations relate to analysing routine electronic patient records, where missing data may be associated with bias and loss of precision [62]. There may also be concerns around the quality of the data entered on databases and variables to adjust for known confounders may not be available [63]. It may be possible to apply specialist techniques to address this [62-64]. For the cross-sectional phases of the study, it will not be possible to conclude temporality of associations.

\section{Dissemination}

Analyses will be disseminated in peer-reviewed manuscripts and through conference proceedings. If requested, analyses will also be prepared as reports or presentations for interested stakeholders. Findings relating directly to clinical care will be fed back to clinical care networks, with a view to informing guideline development.

\section{Conclusions}

There is currently an absence of evidence relating to life expectancy differences in ethnic minority people living with severe mental illnesses such as schizophrenia. In particular, little is known about the experience of cardiovascular disease in these populations and whether there are additional barriers or inequities in service provision. E-CHASM will seek to address these gaps in knowledge through a combination of quantitative analysis of electronic health records and qualitative interviews.

Acknowledgments We are grateful to Ms Sherifat Oduola for providing advice on the 'consent-for-contact' programme as well as information relating to the cohort of people with first episode psychosis. We are grateful to Professor Michael Dewey for advice. We are also grateful to Dr Chin-Kuo Chang and Mr Matthew Broadbent and $\mathrm{Mr}$ Hitesh Shetty for advice on usage of BRC-CRIS data.

\section{Compliance with ethical standards}

Conflicts of interest FG has received honoraria for advisory work and lectures from Roche, Lundbeck, and Sunovion. FG is part of a research team indirectly supported by Janssen and has a family member with professional links to Lilly and GSK. No other conflicts of interest declared. 
Declaration of funding JD is funded by the Health Foundation working with the Academy of Medical Sciences. CM is supported by a European Research Council Consolidator Award (Ref: ERC-CoG2014-Proposal 648837, REACH). RS is funded by the NIHR Specialist Biomedical Research Centre for Mental Health at the South London and Maudsley NHS Foundation Trust and Institute of Psychiatry, King's College London. GT and FG are supported by the National Institute for Health Research (NIHR) Collaboration for Leadership in Applied Health Research and Care South London at King's College London Foundation Trust. GT acknowledges financial support from the Department of Health via the National Institute for Health Research (NIHR) Biomedical Research Centre and Dementia Unit awarded to South London and Maudsley NHS Foundation Trust in partnership with King's College London and King's College Hospital NHS Foundation Trust. GT is supported by the European Union Seventh Framework Programme (FP7/2007-2013) Emerald project. AR is funded by the European Union Horizon 2020 programme OpenMinTeD and KConnect projects, by the National Institute for Health Research (NIHR) Biomedical Research Centre and Dementia Biomedical Research Unit at South London and Maudsley NHS Foundation Trust and King's College London, and by QBurst. The views expressed are those of the author(s) and not necessarily those of the NHS, the NIHR, the Department of Health or any of the funding bodies.

Open Access This article is distributed under the terms of the Creative Commons Attribution 4.0 International License (http://crea tivecommons.org/licenses/by/4.0/), which permits unrestricted use, distribution, and reproduction in any medium, provided you give appropriate credit to the original author(s) and the source, provide a link to the Creative Commons license, and indicate if changes were made.

\section{References}

1. Chang C-K et al (2011) Life expectancy at birth for people with serious mental illness and other major disorders from a Secondary Mental Health Care Case Register in London. PLoS One 6(5): 19590

2. Wahlbeck K et al (2011) Outcomes of nordic mental health systems: life expectancy of patients with mental disorders. Br J Psychiatry 199(6):453-458

3. Lawrence D, Hancock KJ, Kisely S (2013) The gap in life expectancy from preventable physical illness in psychiatric patients in Western Australia: retrospective analysis of population based registers. BMJ 346:f2539

4. Desai M et al (2002) Mental disorders and quality of diabetes care in the veterans health administration. Am J Psychiatry 159:1584-1590

5. Daumit G et al (2006) Adverse events during medical and surgical hospitalizations for persons with schizophrenia. Arch Gen Psychiatry 63:267-272

6. Lawrence DM et al (2003) Death rate from ischaemic heart disease in Western Australian psychiatric patients 1980-1998. Br J Psychiatry 182(1):31-36

7. Taylor DM, McAskill R (2000) Atypical antipsychotics and weight gain-a systematic review. Acta Psychiatr Scand 101(6):416-432

8. Rummel-Kluge C et al (2010) Head-to-head comparisons of metabolic side effects of second generation antipsychotics in the treatment of schizophrenia: a systematic review and meta-analysis. Schizophr Res 123(2-3):225-233
9. Smith $\mathrm{M}$ et al (2008) First-v. second-generation antipsychotics and risk for diabetes in schizophrenia: systematic review and meta-analysis. Br J Psychiatry 192(6):406-411

10. de Leon J, Diaz FJ (2005) A meta-analysis of worldwide studies demonstrates an association between schizophrenia and tobacco smoking behaviors. Schizophr Res 76(2-3):135-157

11. Osborn DJ et al (2007) Relative risk of cardiovascular and cancer mortality in people with severe mental illness from the United Kingdom's General Practice Research Database. Arch Gen Psychiatry $64(2): 242-249$

12. Prince $M$ et al (2007) No health without mental health. Lancet 370(9590):859-877

13. Chang C-K et al (2010) All-cause mortality among people with serious mental illness (SMI), substance use disorders, and depressive disorders in southeast London: a cohort study. BMC Psychiatry 10(1):77

14. Carliner $\mathrm{H}$ et al (2014) Prevalence of cardiovascular risk factors among racial and ethnic minorities with schizophrenia spectrum and bipolar disorders: a critical literature review. Compr Psychiatry 55(2):233-247

15. Bhui $\mathrm{K}$ et al (2003) Ethnic variations in pathways to and use of specialist mental health services in the UK: systematic review. $\mathrm{Br}$ J Psychiatry 182(2):105-116

16. Boydell J et al (2012) Satisfaction with inpatient treatment for first-episode psychosis among different ethnic groups: a report from the UK AESOP study. Int J Soc Psychiatry 58(1):98-105

17. Walkup $\mathbf{J}$ et al (2000) Patients with schizophrenia at risk for excessive antipsychotic dosing. J Clin Psychiatry 61:344-348

18. Diaz F, Leon Jd (2002) Excessive antipsychotic dosing in 2 US state hospitals. J Clin Psychiatry 63:998-1003

19. Segal SP, Bola JR, Watson MA (1996) Race, quality of care, and antipsychotic prescribing practices in psychiatric emergency services. Psychiatr serv 47(3):282-286

20. Pinto $\mathrm{R}$ et al (2010) Differences in the primary care management of patients with psychosis from two ethnic groups: a populationbased cross-sectional study. Fam Pract 27(4):439-446

21. Agyemang $\mathrm{C}$ et al (2009) Cardiovascular disease, diabetes and established risk factors among populations of sub-Saharan African descent in Europe: a literature review. Glob Health 5(7):1-17

22. Creswell JW et al. (2015) Best practices for mixed methods research in the health sciences. Bethesda (Maryland): National Institutes of Health. http://obssr.od.nih.gov/mixed_methods_ research. Accessed 29 July 2015 (August 2011, National Institutes of Health)

23. MacInnes T, P Kenway (2009) London's poverty profile. http:// www.londonspovertyprofile.org.uk/downloads/LondonPovertyPro file.pdf. Accessed 23 June 2015 (City Parochial Foundation and New Policy Institute)

24. Garner S, G Bhattacharyya (2011) Poverty, ethnicity and place. http://www.jrf.org.uk/sites/files/jrf/poverty-ethnicity-place-full.pdf. Accessed 24 July 2015 (in JRF programme paper: Poverty and ethnicity , Joseph Rowntree Foundation)

25. Leeser R (2011) English indices of deprivation 2010: A London perspective, in Intelligence briefing. Greater London Authority, London

26. Fekadu A, Medhin G, Kebede D et al (2015) Excess mortality in severe mental illness: 10-year population-based cohort study in rural Ethiopia. Br J Psychiatry 206(4):289-296

27. Herrett $E$ et al (2010) Validation and validity of diagnoses in the General Practice Research Database: a systematic review. Br J Clin Pharmacol 69(1):4-14

28. Schofield P, Chen R, Crichton N (2012) Methods for assessing cardiovascular disease risk in a UK black population. Heart 98(18):1373-1377 
29. Schofield P, Ashworth M, Jones R (2011) Ethnic isolation and psychosis: re-examining the ethnic density effect. Psychol Med 41(06): 1263-1269

30. Mathur R, Pollara E, Hull S et al (2013) Ethnicity and stroke risk in patients with atrial fibrillation. Heart 99:1087-1092

31. Read Codes Version 3. http://systems.hscic.gov.uk/data/uktc/ readcodes. Accessed 1 Feb 2016

32. Noble $M$ et al (2006) Measuring multiple deprivation at the small-area level. Environ Plan A 38(1):169-185

33. World Health Organisation (1978) Mental disorders: glossary and guide to their classification in accordance with the ninth revision of the international classification of diseases. WHO, Geneva

34. Nazareth I et al (1993) Accuracy of diagnosis of psychosis on general practice computer system. BMJ 307(6895):32-34

35. Hardoon $\mathrm{S}$ et al (2013) Recording of severe mental illness in United Kingdom Primary Care, 2000-2010. PLoS One 8(12):e82365

36. (2015) British National Formulary (BNF), vol 70. BMJ Publishing Group Ltd and Royal Pharmaceutical Society of Great Britain

37. Stewart R, Soremekun M, Perera G et al (2009) The South London and Maudsley NHS Foundation Trust Biomedical Research Centre (SLAM BRC) case register: development and descriptive data. BMC Psychiatry 9(51). doi:10.1186/1471-244X-9-51

38. Kadra G et al (2015) Extracting antipsychotic polypharmacy data from electronic health records: developing and evaluating a novel process. BMC Psychiatry 15(1):166

39. World Health Organisation (1993) The ICD-10 classification of mental and behavioural disorders: diagnostic criteria for research. WHO, Geneva

40. Murdoch TB, Detsky AS (2013) The inevitable application of big data to health care. JAMA 309(13):1351-1352

41. Meystre SM et al (2008) Extracting information from textual documents in the electronic health record: a review of recent research. IMIA Yearbook 2008: access to health. Information 3(1):128-144

42. Su Y-P et al (2014) Mini-mental state examination as a predictor of mortality among older people referred to secondary mental healthcare. PLoS One 9(9):e105312

43. Wu C-Y et al (2013) Evaluation of smoking status identification using electronic health records and open-text information in a large mental health case register. PLoS One 8(9):e74262

44. Patel R et al (2015) Negative symptoms in schizophrenia: a study in a large clinical sample of patients using a novel automated method. BMJ Open 5:e007619

45. Cunningham $\mathrm{H}$ et al (2013) Getting more out of biomedical documents with GATE's full lifecycle open source text analytics. PLoS Comput Biol 9(2):e1002854

46. Galobardes B et al (2006) Indicators of socioeconomic position (part 1). J Epidemiol Community Health 60(1):7-12

47. Chandola T, Jenkinson C (1999) Social class differences in morbidity using the New UK. National statistics socio-economic classification: do class differences in employment relations explain class differences in health? Ann N Y Acad Sci 896(1):313-315

48. Hippisley-Cox J, Coupland C, Robson J, Brindle P (2010) Derivation, validation, and evaluation of a new QRISK model to estimate lifetime risk of cardiovascular disease: cohort study using QResearch database. BMJ 341:c6624. doi:10.1136/bmj. c6624

49. Carrà G et al (2014) The prevalence of metabolic syndrome in people with severe mental illness: a mediation analysis. Soc Psychiatry Psychiatr Epidemiol 49(11):1739-1746

50. Bourque F (2015) A mixed methods study of the relation between migration, ethnicity and psychosis, in Institute of Psychiatry, Psychology and Neuroscience. King's College London, London

51. Mallett R et al (2002) Social environment, ethnicity and schizophrenia. Soc Psychiatry Psychiatr Epidemiol 37(7):329-335

52. Braun V, Clarke V (2013) Successful qualitative research. Sage Publications Ltd., London

53. NVivo qualitative data analysis Software; QSR International Pty Ltd. Version 10

54. O'Cathain A, Murphy E, Nicholl J (2010) Three techniques for integrating data in mixed methods studies. BMJ 341:c4587. doi:10.1136/bmj.c4587

55. Fernandes AC et al (2013) Development and evaluation of a deidentification procedure for a case register sourced from mental health electronic records. BMC Med Inform Decis Mak 13:71

56. Mathur R et al (2012) Ethnic differences in primary care management of diabetes and cardiovascular disease in people with serious mental illness. Br J Gen Pract 62(601):e582-e588

57. Bhopal R et al (1999) Heterogeneity of coronary heart disease risk factors in Indian, Pakistani, Bangladeshi, and European origin populations: cross sectional study. BMJ 319(7204):215-220

58. Becker E et al (2006) Health survey for England: 2004. In: Sproston K, Mindell J (eds) The health of minority ethnic groups, in health survey for England. The information centre, Leeds

59. Agyemang $C$ et al (2011) Diabetes prevalence in populations of South Asian Indian and African Origins: a comparison of England and The Netherlands. Epidemiology 22(4):563-567. doi:10. 1097/EDE.0b013e31821d1096

60. Kirkbride JB et al. (2012) Systematic review of the incidence and prevalence of schizophrenia and other psychoses in England. http:// www.psychiatry.cam.ac.uk/files/2014/05/Final-report-v1.05-Jan12.pdf. Accessed 22 Sept 2015. (Department of health policy research programme. $\mathrm{p} 846$ )

61. Bhopal RS (2012) Research agenda for tackling inequalities related to migration and ethnicity in Europe. J Public Health 34(2):167-173

62. Sterne JAC, White IR, Carlin JB et al (2009) Multiple imputation for missing data in epidemiological and clinical research: potential and pitfalls. BMJ 338:b2393. doi:10.1136/bmj.b2393

63. Stuart EA, DuGoff E, Abrams M, Salkever D, Steinwachs D (2013) Estimating causal effects in observational studies using electronic health data: challenges and (some) solutions. eGEMs (Generating Evidence \& Methods to improve patient outcomes) 1(3), article 4. doi:10.13063/2327-9214.1038

64. Welch C, Bartlett J, Petersen I (2014) Application of multiple imputation using the two-fold fully conditional specification algorithm in longitudinal clinical data. Stata J 14(2):418-431 